\title{
Féeries
}

Études sur le conte merveilleuX, XVII $-\mathrm{XIX}{ }^{\mathrm{e}}$ siècle

\section{Le savoir d'un conte moins conte que les autres}

Le «Sans Parangon » de Préchac et les limites de l'absolutisme

The Knowledge of a Tale less a Tale than Others: Préchac's "Sans Parangon" and the Limits of Absolutism

\section{Hall Bjørnstad}

\section{OpenEdition \\ Journals}

Édition électronique

URL : http://journals.openedition.org/feeries/711

DOI : 10.4000/feeries.711

ISSN : 1957-7753

Éditeur

UGA Éditions/Université Grenoble Alpes

Édition imprimée

Date de publication : 1 juillet 2009

Pagination : 163-178

ISBN : 978-2-84310-140-3

ISSN : $1766-2842$

Référence électronique

Hall Bjørnstad, «Le savoir d'un conte moins conte que les autres », Féeries [En ligne], 6 | 2009, mis en ligne le 15 septembre 2010, consulté le 08 septembre 2020. URL : http://journals.openedition.org/ feeries/711; DOI : https://doi.org/10.4000/feeries.711 


\title{
LE SAVOIR D'UN CONTE MOINS CONTE QUE LES AUTRES
}

\author{
LE « SANS PARANGON » DE PRÉCHAC ET LES LIMITES \\ DE L'ABSOLUTISME
}

\begin{abstract}
0 ANS DOUTE LA CRITIQUE MODERNE A-T-ELLE RAISON de souligner le risque que court le conte encomiastique de devenir trop conte - rabaissant par là la noblesse et la vérité des exploits du héros loué - et de montrer comment ce souci est inscrit tout au long du texte de "Sans Parangon » de Jean de Préchac, dès le titre du recueil dont il est issu (Contes moins contes que les autres, I698) et le nom même du héros éponyme, jusqu'au dénouement de l'histoire, quand Sans Parangon se prépare à faire la guerre aux fées ${ }^{\mathrm{I}}$. Mais ce conte à visée politique court aussi un autre risque, d'ordre inverse : devenir trop vrai. Retraçant allégoriquement les grandes étapes du règne de Louis XIV comme étant structurées selon les caprices et les défis lancés au prince par la princesse Belle-Gloire, le
\end{abstract}

I. Pour la critique récente, voir Anne Defrance, "Le conte de fées au risque de l'éloge politique. La tyrannie des fées détruite ( $\mathrm{M}^{\mathrm{me}} \mathrm{d}$ 'Auneuil) et autres contes de la première génération ", dans Le conte merveilleux au XVIII ${ }^{e}$ siècle. Une poétique expérimentale, textes réunis et présentés par Régine Jomand-Baudry et Jean-François Perrin, Paris, Kimé, 2002, p. 55-73; Teresa Di Scanno, "Monsieur de Préchac ou l'allégorie courtisane ", dans Les contes de fées à l'époque classique (1680I715), Naples, Liguori, 1975, p. I63-170 ; Françoise Gevrey, Introduction, dans Contes moins contes que les autres, précédés de L'Illustre Parisienne, éd. Gevrey, Paris, STFM, I993, p. I-XxxviII ; Tony Gheeraert, Introduction [à la section sur Contes moins contes que les autres], dans Perrault, Fénelon, Mailly, Préchac, Choisy et anonymes, Contes merveilleux, éd. Gheeraert, Paris, Champion, 2005, p. 665-686 ; Éric Méchoulan, «Le pouvoir féerique », dans Féeries, n ${ }^{\circ}$, Politique du conte, 2006. Dans ce qui suit, l'édition de référence sera celle de Gheeraert (ouvr. cité, p. 687-729), et les citations seront suivies directement du numéro de page entre parenthèses. Gheeraert modernise systématiquement l'orthographe de l'original, un principe qui a été suivi ici aussi pour les citations d'autres œuvres du XVII ${ }^{\mathrm{e}}$ siècle. 
texte n'est pas loin de suggérer que les exploits royaux sont dépourvus de tout fondement rationnel. Ainsi, quand Belle-Gloire lance au prince Sans Parangon, vers la fin du conte, l'avertissement suivant : " tes trésors sont épuisés par les complaisances que tu as eues pour moi » (p. 722), ses paroles ressemblent fort à celles de Fénelon dans sa fameuse lettre adressée au roi Sans Parangon réel, cinq ans avant la parution du conte : "Vous avez passé votre vie entière hors du chemin de la vérité et de la justice ", le malheur actuel n'étant que la conséquence funeste " de cette guerre de I672, entreprise pour votre gloire ${ }^{2} »$.

Le présent article est fondé sur l'intuition que les deux risques évoqués ci-dessus le sont exactement dans la mesure où ils exposent un savoir potentiellement dangereux sur les limites de la logique qui sous-tend l'absolutisme. Si, comme l'indique Louis Marin, c'est « dans l'essence de tout pouvoir de tendre à l'absolu ${ }^{3}$ ", la gloire devient la figure essentielle d'un absolutisme qui tend vers ses limites et sa personnification un lieu privilégié pour l'étude de cette logique. Une telle approche implique une réorientation de la perspective de la critique récente, qui, elle, se penche plutôt sur l'étrangeté du conte de Préchac en mesurant, d'une part, l'écart entre celui-ci et le genre du conte de fées en général, et, d'autre part, l'écart entre sa version de l'histoire française du règne de Louis XIV et les réalités historiques souvent plus sinistres. L'ambition ici est donc de suggérer que par l'examen de l'étrange logique à l'œuvre dans "Sans Parangon ", on parviendra non seulement à une compréhension plus fine de la démarche encomiastique, mais aussi, peut-être, à une meilleure perception du contexte absolutiste lui-même, et de l'excès qui s'y inscrit sous le nom de "gloire".

\section{II}

Le pluriel du terme "Contes " dans le titre du livre de Préchac ne renvoie qu’à deux éléments : "Sans Parangon ", suivi de "La reine des fées ". La dédicace qui ouvre le recueil donne le ton des rapports entre royauté et féerie auxquels nous nous intéressons ici. À qui dédier l'éloge du roi qui va suivre, alors que le genre choisi pour le faire est trop bas pour le dédier

2. Citée ici d'après Joël Cornette, Le roi de guerre : Essai sur la souveraineté dans la France du Grand Siècle, Paris, Payot, 2000 [1993], p. 420, n. 39. La lettre n’a sans doute jamais été lue par le roi.

3. Louis Marin, Le portrait du roi, Paris, Minuit, I98I, p. I2. 
au roi lui-même? Préchac résout le problème en s'adressant à « la Sans Parangon des cascades " - "la très haute, très magnifique, et excellentissime cascade [du château royal] de Marly » - plutôt qu'au Sans Parangon des rois :

\section{Charmante cascade,}

Le rapport qu'il y a de vous aux ouvrages des fées, m’engage à vous dédier ces contes, qui paraîtront moins fabuleux toutes les fois qu'on examinera avec attention votre surprenante beauté, et tous les autres prodiges dont vous êtes environnée, cet agréable château où l'on trouve toutes choses sans y rien apporter, ces jardins délicieux, ce superbe bocage, qui sans s'assujettir à l'ordre des saison, ni sans attendre le secours des siècles, est devenu une vaste forêt ; toutes ces merveilles justifient mes contes. (p. 687)

On trouve dès la première phrase une juxtaposition souriante des " ouvrages " du roi (la cascade, le château, les jardins...) et de ceux des fées. Il convient cependant de noter que la description en termes du merveilleux de la demeure privée du roi, et surtout de la grande cascade, n'est aucunement l'invention de Préchac, comme en témoigne la Princesse Palatine quand elle écrit dans une lettre : "On dirait que ce sont des fées qui travaillent $\mathrm{ici}^{4}$. " La mobilisation d'un vocabulaire de l'extraordinaire ("votre surprenante beauté ", "tous les autres prodiges", " toutes ces merveilles» - et plus loin dans la dédicace : «bien loin de craindre le soleil qui tarit d'ordinaire les autres sources ", " une abondance prodigieuse ", "la Sans Parangon des cascades" (ibid.) ) $)^{5}$ veut faire croire à une réalité royale qui dépasse la féerie. De la sorte, « ces contes [...] paraîtront moins fabuleux » pour deux raisons précises. D’abord une raison d'ordre général : les ouvrages des fées sont moins fabuleux que ceux du roi (tels que ceux-ci sont visibles dans le monde réel, par exemple dans la cascade). Ensuite, dans ce cas spécifique, on est en présence d'un conte allégorique qui fait le récit, dans un code très facilement déchiffrable, des exploits réels du roi. $\mathrm{Si}$, comme le souligne la dernière phrase de la dédicace, « la Sans Parangon des cascades [...] mérit[e] toutes les louanges et tous les applaudissements qu'on saurait [lui] donner " (ibid.), la même chose vaut évidemment, et même à plus forte raison, pour le Sans Parangon royal. La dédicace se clôt

4. Cité d'après Gevrey, art. cité, p. xxix. Pour une description détaillée de la cascade de Marly, « une chute d'eau artistement décorée de portiques et de rocailles, et qui se déversait du haut de la colline sur cinquante-deux bassins de marbre rose ", y compris des défis techniques impliqués dans sa conception et sa construction, on consultera Gheeraert, art. cité, p. 67I-673.

5. C'est moi qui souligne. 
ainsi sur la glorification qui est due au roi, tandis que le conte principal du recueil le glorifie en donnant à voir l'anatomie de cette gloire.

Allons un peu plus loin dans l'examen du geste dédicatoire de Préchac. Au seuil de son ouvrage, l'auteur soulève " [l]e rapport qu'il y a de vous [la cascade ; les ouvrages extraordinaires du roi] aux ouvrages des fées". Le sien propre semble se situer quelque part au milieu de ces deux catégories, louant et applaudissant les ouvrages du roi, en les introduisant dans un cadre féerique. Or, puisque le pouvoir magique des fées n'existe guère sans l'œuvre du conteur, ce rapprochement pourrait inviter à se poser des questions sur le statut ontologique de la gloire royale : ne passe-t-on pas ici d'un modèle épico-mythique (une gloire intrinsèque, fondée sur les vertus du roi, qui devient extrinsèque à travers ses exploits glorieux) ou théologico-politique (la glorification d'un roi-dieu qui n'en a aucunement besoin), à un modèle de fiction pure (une glorification qui crée son objet, qui lui est consubstantielle) ? On verra par la suite comment le conte sur le prince Sans Parangon résiste à un tel dérapage conceptuel, dérapage néanmoins inscrit dans sa structure narrative. Mais il faut dès à présent insister sur la présence de cette tension hors du sous-genre du conte encomiastique. On en retrouve en fait une formulation explicite dans un énoncé de Louis XIV lui-même, qui aurait, selon Charles Perrault, interpellé les artisans de sa gloire dans la "Petite Académie " de la manière suivante :

Vous pouvez, Messieurs, juger de l'estime que je fais de vous, puisque je vous confie la chose du monde qui m’est la plus précieuse, qui est ma gloire : je suis sûr que vous ferez des merveilles; je tâcherai de ma part de vous fournir de la matière qui mérite d'être mise en ouvre par des gens aussi habiles que vous êtes ${ }^{6}$.

La fonction de la littérature encomiastique officielle serait donc de trouver une forme qui convienne à la matière glorieuse fournie par le roi. Le choix des mots du roi, rapporté par un écrivain qui allait lui aussi écrire des contes par la suite, n'est pas innocent dans notre contexte : faire des merveilles en faisant faire au roi des merveilles. Telle est aussi l'ambition de notre écrivain, dont l'habileté est certes un peu moins validée par le

6. Mémoires de Ch. Perrault, dans Euvres choisies de Ch. Perrault, avec les mémoires de l'auteur..., éd. Collin de Plancy, Paris, Brissot-Thivars, I826, p. Xxv-Xxvi ; c'est moi qui souligne. L'énoncé était adressé à la " Petite Académie " (l'Académie royale des Inscriptions et Médailles), qui travaillait à l'histoire du roi, très tôt dans le règne personnel de celui-ci. L'expression « les artisans de sa gloire » fait évidemment référence à l'ouvrage classique d'Orest Ranum, Artisans of Glory: Writers and Historical Thought in Seventeenth-Century France, Chapel Hill, University of North Carolina Press, I980. 
pouvoir, Jean de Préchac. Car, nous venons de l'entendre, ce sont « toutes $[1]$ es merveilles " procurées par le roi qui « justifient $\left[\right.$ s]es contes ${ }^{7}$ ».

" Il y avait une fois un roi et une reine qui menaient une vie fort particulière... " (p. 689). Dès la première phrase, à la première ligne du conte, Préchac fonde son projet de panégyrique sur une solidarité entre royauté et féerie qui préexiste à son texte. Les phrases qui suivent traduisent explicitement le lien privilégié, quoique tendu, unissant les grands et les fées : un roi qui "mépris[e] leurs enchantements ", des "fées ... fort irritées " (ibid.), et une reine médiatrice qui consulte les fées, mais à l'insu du pouvoir mâle. Dès le seuil du texte, s'établit ainsi la superposition d'un monde magique secret et de l'exercice positif du pouvoir, superposition qui va, par la suite, donner lieu à la narration féerisée des exploits de Louis XIV hors du texte.

Le héros éponyme du conte n'est que lentement mis en scène. Le roi et la reine de la première phrase sont en fait ses grands-parents maternels (alias Philippe III d'Espagne et Marguerite d'Autriche), et il faut attendre le tiers du conte (I3 pages sur 40 dans l'édition de référence) pour arriver à la naissance effective du prince Sans Parangon lui-même.

À ce moment, beaucoup est déjà joué. La mère de Sans Parangon, la reine Belle-Main (alias Anne d'Autriche) a pu mobiliser une bonne fée, Clairance, pour compenser l'influence néfaste de la fée irritée, la méchante Ligourde. Dès lors, l'avenir du jeune prince se joue à travers les sorts et les contre-sorts des deux fées. La longue attente avant sa naissance était déjà due à l'intervention de Clairance, qui, pour prévenir les intentions

7. On consultera les introductions de Gevrey (art. cité, p. IV-IX) et de Gheeraert (art. cité, p. 666-669) pour une présentation détaillée des liens entre Préchac et le pouvoir royal. Il suffit ici de noter que, bénéficiant de la faveur de Monsieur, le frère du roi, à partir de sa désignation comme lecteur de celui-ci en I676, Préchac mène une existence de courtisan et de romancier entre I677 et I690, publiant une trentaine d'œuvres de fiction ; en effet, comme le souligne Gheeraert, les « deux carrières de Préchac [...] sont inséparables : il ne fut auteur que pour flatter les grands et ainsi mieux faire sa cour " (ibid., p. 667-668). Nous ignorons pourquoi Préchac reprend sa plume après huit ans de silence en I698, publiant les Contes moins contes que les autres, ses seuls contes de fées. Comme l'indique Mary Elizabeth Storer, « à en croire l'auteur, ses divers ouvrages eurent du succès auprès des personnes les plus importantes de la cour ", y compris le roi lui-même ( $L a$ mode des contes de fées (I685-I700), Paris, Champion, I928, p. I8I), d'où la spéculation que voici : "on peut très bien supposer qu'il [Louis XIV] lut et goûta même Sans Parangon, cette allégorie féerique de sa Belle-Gloire » (ibid.). 
violentes de Ligourde, l'avait enlevé après une première naissance invisible, le gardant pendant 2I ans dans un monde enchanté. Ce hiatus permet d'expliquer non seulement la longue stérilité d'Anne d'Autriche avant la naissance de Louis Dieudonné, mais aussi la précocité politique du jeune prince Sans Parangon, car Clairance fait en sorte qu'il reçoive, avant sa véritable naissance, une solide instruction dans l'art de régner, l'art de la guerre, les beaux arts, etc. Autrement dit, le prince reçoit chez Clairance une pré-éducation, dont il se souviendra une fois que commence sa formation réelle, après sa deuxième naissance : "il apprenait facilement toutes choses, se souvenant bien qu'il les avait déjà sues " (p. 702). La même chose vaut en fait pour son amour de Belle Gloire, " la princesse de la Chine, qui était sans contredit la plus belle et à même temps la plus fière princesse de la terre, et qui était enchantée pour plusieurs siècles " (p. 696-697). Dès les premiers jours de son enlèvement " prénatal ", le prince est sous le charme des chants de la princesse, et bientôt «Belle Gloire s'acquit un si furieux ascendant sur son esprit, qu'il s'ennuyait toujours partout où il ne la voyait pas ; [...] ne se proposant en toutes choses que de plaire à Belle Gloire » (p. 698). Ainsi le prologue du conte consiste-il en une longue prolepse : le jeu amoureux des défis lancés par la fière princesse, puis la réalisation des défis par le vaillant prince, cette dynamique qui structure les deux tiers du conte sur la vie de Sans Parangon après sa naissance (soit le récit féerisé des exploits de Louis XIV) ne fait que reprendre pareil jeu pratiqué avant sa naissance.

D'un point de vue encomiastique, il existe un danger que trop ne soit joué avant la naissance du prince. En effet, pour efficace que soit la prolepse éducative d'un point de vue narratologique, ce procédé risque néanmoins de limiter le potentiel héroïque du prince, en soumettant son pouvoir à celui des fées. Ou pire, peut-être est-ce cette efficacité narratologique ellemême qui pose problème, en limitant la liberté absolue du héros. Avant d'examiner la narration de la vie « réelle » du prince Sans Parangon, il n'est donc pas sans intérêt de regarder comment celle de son existence " prénatale » sert, à plusieurs reprises, à miner la supériorité absolue des fées dans leur propre monde enchanté.

Considérons d'abord la toute première phrase, fort longue, qui décrit l'enlèvement du prince par la bonne fée :

Clairance enleva Sans Parangon; et comme son art lui apprenait les grandes choses que ce prince opérerait à l'avenir, elle se fit un grand plaisir de le bien élever; elle eut une attention particulière à lui préparer un appartement très propre et fort sain ; et comme la fée savait que les enfants tiennent souvent de leurs nourrices, elle lui choisit pour le nourrir une reine enchantée, qui était de bon tempérament, et avait les 
inclinations fort nobles ; plusieurs grâces et amours enchantés eurent ordre de bercer l'enfant ; il me serait aisé de faire une description de son berceau et de ses langes, mais on n'a qu'à imaginer tout ce qu'il y peut avoir de plus riche, et de meilleur goût dans un palais enchanté, et cela se trouvera encore fort au-dessous du berceau et des langes de Sans Parangon. (p. 696 ; c'est moi qui souligne)

L'« art » de Clairance évoqué dans le premier passage souligné est celui de la voyance, ce qui n'est pas sans faire réfléchir au sous-entendu de la suite de la phrase. Le fait d'avoir été si bien élevé par Clairance devait expliquer la précocité et le triomphe général du prince dans la vie (c'est la fonction de la prolepse). Mais en réalité la fée n'entreprend son projet d'éducation qu'à cause de l'avenir lumineux du prince. Comme si l'avenir de ce dernier était fixé ou du moins existait indépendamment de l'éducation de Clairance, sans que celle-ci fût nécessaire.

Pareil trouble apparaît dans les dernières lignes de la même phrase, à travers la description, ou plutôt la non-description, du berceau et des langes du jeune prince. Ce deuxième passage souligné dans la citation ci-dessus a été perçu comme une "ellipse cavalière d'informations que le romancier ne daigne pas fournir ${ }^{8} »$. Il suffit pourtant d'observer la contradiction entre l'aisance initiale et l'impossibilité qui suit, pour voir que la situation est plus complexe : l'imagination aisée dont il est ici question porte en fait sur quelque chose qui est fort au-dessus de « tout ce qu'il y peut avoir de plus riche, et de meilleur goût dans un palais enchanté » (c'est moi qui souligne) - et cela à l'intérieur même d'un palais enchanté. Si Clairance transcende ainsi la richesse et le goût du monde féerique, c'est sans doute que son ouvrage (le berceau et les langes) se crée sous l'influence des " grandes choses que ce prince opérerait à l'avenir » dans le monde réel.

L'exemple le plus frappant de ce déplacement dans la hiérarchie des pouvoirs - celui des fées et celui du prince, et cela déjà pendant le séjour prénatal de ce dernier chez Clairance -, se rencontre quelques pages plus loin. L'enjeu ici n'est plus la description faite par le narrateur, ni l'imagination du lecteur, mais bien le pouvoir d'imagination du prince lui-même. Pourvu par la bonne fée d'une baguette magique "dont il n'avait qu'à frapper trois fois pour faire paraître tout ce qu'il imaginait ", Sans Parangon fait jouer sa maîtrise des beaux-arts, en construisant et

8. Jacques Chupeau, cité par Gheeraert, ouvr. cité, p. 696, n. I. Voir aussi Gevrey, art. cité, p. Xxx, selon laquelle "Préchac fait [ici] l'économie des descriptions". Les trois critiques s'arrêtent sur ces lignes comme caractéristique du style de Préchac, sans considérer son éventuelle complexité sémantique. 
ornant un "superbe palais ", qui n'est pas sans rappeler celui de Versailles. Après la description des escaliers et des appartements, et avant celle du jardin, on trouve le passage que voici :

[...] on passait ensuite dans une grande galerie ornée de glaces et de belles statues de marbre et de bronze, avec des peintures merveilleuses, où l'on remarquait des actions d'un héros si prodigieuses, qu'on ne voyait rien de pareil même dans la fable. (p. 699)

La description allégorique de Versailles est ici encore plus facilement déchiffrable qu'ailleurs, puisque précisément la galerie des Glaces portait autrefois le nom de "grande galerie ». De qui cette galerie féerisée et enchantée est-elle l'œuvre ? L'exécution du palais et de la galerie est, certes, opérée par Clairance, mais l'imagination, la conception même sont l'œuvre du futur roi, qui anticipe ainsi sur l'ouvrage de son architecte, de ses artistes et de son peintre (alias Le Brun), tout comme sur ses propres actions héroïques, figurées dans l'ouvrage de ce dernier. Et l'on reconnaitra dans le passage ci-dessus la distinction évoquée par Louis XIV devant la Petite Académie, entre la matière fournie par le roi et les merveilles qu'en feraient les académiciens, tandis qu'ici sont évoquées " des peintures merveilleuses » et "des actions [...] prodigieuses ». Lordre est juste inversé, puisque dans le conte la représentation précède les actions, le prince jouant aussi bien le rôle du futur héros que celui de l'artiste actuel.

Si donc cette grande galerie enchantée donne à voir des choses si extraordinaires "qu'on ne vo[it] rien de pareil même dans la fable ", c'est moins dû au pouvoir d'exécution de la fée (puisqu'en l'occurrence on voit bien des merveilles dans la fable) qu'au pouvoir d'imagination et d'anticipation du futur roi. Ce sont donc les images des actions héroïques à venir qui dépassent la fable, les mêmes actions qui seront racontées dans le reste du conte de Préchac. Dans son existence "prénatale ", le prince produit ainsi sa propre prolepse, qui dépasse celle du conte que nous sommes en train de lire.

\section{IV}

Ces trois exemples d'un certain trouble dans la souveraineté des fées, au sein même du monde enchanté où Sans Parangon reçoit son initiation politique "prénatale ", annoncent un déplacement du merveilleux après la naissance du prince. Les fées seront presque absentes du royaume de Sans Parangon, sauf dans le cas des visites régulières de la princesse Belle Gloire. Mais cette dernière n'est visible que pour Sans Parangon. Belle 
Gloire domine le prince, mais de manière invisible, intériorisée, exerçant son influence sur sa volonté hors la vue de ses sujets, et à leur insu. Ainsi, Sans Parangon est étrangement situé à la frontière des deux mondes : il incarne un pouvoir mâle et souverain, tout en restant ouvert à une réalité enchantée féminine, inaccessible à ses prédécesseurs masculins évoqués dans le conte (notamment son père ${ }^{9}$ et son grand-père maternel, alias Louis XIII et Philippe III d'Espagne). Comme si la porosité entre ces deux réalités était nécessaire pour lui permettre de réaliser son potentiel extraordinaire et le rendre digne de son nom, nom qui lui a d'ailleurs été accordé par la bonne fée " parce que jamais prince ne pourra lui être comparé » (p. 696). Les exploits prodigieux de Sans Parangon sont d'autant plus merveilleux qu'ils s'achèvent souverainement, sans participation magique des fées, dans un monde qui est comme désenchanté, hormis bien sûr la magie nouvellement acquise du roi. Et pourtant, même si le pouvoir du roi est absolu, même s'il aurait bien $p u$ en faire autant sans l'intervention secrète de Belle Gloire, le conte ne laisse planer aucun doute sur le fait qu'il n'aurait pas voulu le faire, qu'il ne l'aurait pas fait effectivement sans elle.

Pour un bon exemple du dynamisme à l'œuvre dans la vie héroïque de Sans Parangon, il suffit de retourner à la construction de sa demeure royale, dans sa version non-enchantée cette fois. Après une première demande de la Princesse désirant un beau palais où le prince puisse la recevoir,

[...] il fit bâtir dans la capitale de ses États, un des plus beaux palais du monde, avec des jardins très agréables et proportionnés à la magnificence du palais. Ce grand ouvrage était presque fini, lorsque Belle Gloire étant allée visiter le prince à son ordinaire, elle lui fit connaître qu'elle n'aimait point le séjour des villes, et que s'il voulait lui donner un témoignage bien véritable de son attachement, et de sa complaisance pour elle, il fallait lui bâtir à la campagne, un palais et des jardins semblables à ceux qu'il avait imaginés lui-même chez Clairance, par la vertu de sa baguette (p. 710).

Après le Louvre, voici donc le château de Versailles. Or, on le sait, « le palais de la fée n'était qu'une illusion ", et le pauvre Sans Parangon se trouve " épouvanté d'une proposition si extravagante " (ibid.), et rempli d'un " embarras [sans] pareil " face à "l'impossibilité [qu'il trouvait] à l'exécution de ce grand dessein » (p. 7II). Belle Gloire ne fait pourtant que renforcer le défi :

9. Père dont le lecteur n'est pas surpris de savoir qu'il est « mort pendant que Sans Parangon était encore bien jeune " (p. 703). 
Tu sais bien, reprit Belle Gloire, que les choses ordinaires ne m'accommodent point, et que je n'aime que celles qui approchent de l'impossible ; je t'ai fait connaître ce que je désire, c'est à toi à te consulter, et à examiner si tu as, et assez de courage, et assez d'envie de me plaire, pour l'entreprendre. (p. 7IO)

À sa propre surprise, Sans Parangon se montre à la hauteur du défi. En effet,

[il] n'eut jamais de repos que le palais et les jardins ne fussent dans leur perfection

[...] et quoiqu'il ne tâchât qu’à imiter ce qu'il avait déjà fait chez la fée, il est constant qu'il surpassa le palais enchanté en beaucoup de choses (p. 7II).

Les dernières lignes marquent un moment important dans la réorientation du merveilleux à des fins encomiastiques : la réalité royale supplante l'illusion féerique, le palais réel surpasse le palais enchanté. Les vraies merveilles ne se rencontrent pas dans les contes de fées, mais à Versailles.

Il serait tentant d'ajouter : quod erat demonstrandum. Ce conte est "moins conte que les autres" puisqu'il entend nous présenter la vérité allégorique de Versailles et de son roi. Il faut pourtant noter que le palais enchanté était déjà l'œuvre du roi, qui ne fait ici qu' "imiter ce qu'il avait déjà fait chez la fée ». En surpassant le palais enchanté, c'est donc son propre exemple qu'il dépasse, restant de la sorte fidèle à son propre nom, au plus haut degré. Autrement dit, Sans Parangon dépasse même son propre exemple magique...

La logique qui se dessine ici n'est point celle d'un mouvement qui tend vers le repos, la paix satisfaite, laquelle serait atteinte une fois la démonstration parvenue à sa fin, c'est-à-dire une fois que Versailles aura atteint sa perfection définitive, comme pourrait le laisser deviner la citation cidessus : il "n'eut jamais de repos que... ". On est plutôt ici en présence d'un mouvement qui tend vers le dépassement héroïque et perpétuel, vers l'extrême, l'excès. Et par là, inévitablement, vers son propre épuisement. Sa figure est celle de la gloire, ici personnifiée sous le nom de «Belle Gloire ", qui désire, nous venons de le lire, l'extraordinaire et l'impossible. Il est temps à présent de s'interroger sur la fonction de Belle Gloire dans le conte : quelle place occupe-t-elle dans la structure globale, et quelle est son interprétation allégorique?

\section{V}

Le déroulement de la construction féerisée de Versailles est typique de la narration des actions héroïques de Sans Parangon. D’abord le défi malaisé 
lancé par la princesse enchantée, puis l'étonnement du roi devant un tel défi, enfin la réussite tout aussi surprenante que triomphale du roi. Tout naturellement, chaque nouveau déf lancé par Belle Gloire conduit Sans Parangon à un exploit héroïque dont le corrélat (plus ou moins héroïque...) dans la vie de Louis XIV, est facilement identifiable pour le lecteur averti : la campagne de Franche-Comté (I668), les travaux du Canal des Deux Mers (I666-I68I), le passage du Rhin (I672), une série de victoires spécifiques dans la guerre de la Ligue d'Augsbourg (I688-I697), etc. ${ }^{\text {Io }}$

L'examen approfondi de la mise en ouvre de cette dynamique qui structure les derniers deux tiers du conte met en évidence deux traits qui, de prime abord, pourraient être jugés accidentels, mais dont la récurrence systématique signale la valeur essentielle. Il s'agit premièrement de l'insistance de Belle Gloire sur la dignité, la sienne propre et celle de Sans Parangon, les deux étant de toute façon étroitement liées. Sans Parangon doit se rendre digne de Belle Gloire (p. 707, 712, 713, 723, 724), mais aussi être digne de lui-même, tantôt en honorant son nom (p. 707, 719, 726,727 ), tantôt en se détournant de ceux qui sont indignes de lui, qu'il s'agisse d'ennemis (p. 718) ou de maîtresses (p. 70I).

La deuxième remarque porte sur le stade intermédiaire dans la dynamique du défi, à savoir la réaction à celui-ci telle qu'elle est exprimée par le roi - ou tout simplement par le narrateur. Nous savons déjà que la princesse n'aime que les choses " qui approchent de l'impossible» (p. 7IO). Au-delà de l'insistance sur cette impossibilité, il y a une autre qualification qui revient sans cesse, consistant à souligner dans quelle mesure les défis de Belle Gloire sont perçus comme des défis au bon sens : " [c]e terrible discours " (p. 708), " une proposition si extravagante " (p. 710), " une entreprise si nouvelle et si hardie" (p. 713), et finalement " ce ridicule projet" (p. 725).

Les deux caractéristiques qui viennent d'être évoquées sont bien des traits essentiels du mouvement que nous tentons de cerner. Intéressonsnous à présent à leur coïncidence, soit la rencontre discursive entre l'insistance sur la dignité, et celle sur l'extravagance des défis. Une telle rencontre se produit vers la fin du conte. C'est en fait elle qui marque la transition vers l'étrange dénouement où Belle Gloire présente à Sans Parangon son défi final, l'invitant à « faire la guerre aux fées» (p. 727). La

Io. Pour le lecteur de l'époque, le déchiffrement de l'allégorie participait sans doute pour une part importante au plaisir de la lecture. Pour le lecteur moderne un peu moins averti, il existe l'excellente annotation des éditions de Gevrey et de Gheeraert. 
princesse affirme que ce défi trouve son origine dans les exploits héroïques du prince. Elle lui fait connaître que :

son grand courage et les choses extraordinaires qu'elle lui avait vu faire, lui avaient inspiré une pensée qui paraîtrait extravagante, mais qu'elle trouvait digne de Sans Parangon. (Ibid.)

Extraordinaire, impossible, extravagant, ridicule : nous devinons dans les mots de la princesse enchantée un glissement sur l'échelle suggérée par ces adjectifs. Les actions extraordinaires du prince inspirent à la princesse une pensée qui sans doute paraîtra extravagante aux autres, mais qui est digne de lui selon elle. S'agirait-il justement d'une pensée qui est digne parce qu'elle défie le bon sens, parce qu'elle défie la logique ordinaire et rationnelle ? La dignité glorieuse de cette pensée extravagante résidet-elle précisément dans l'appropriation définitive de l'art de faire des merveilles? Nous trouvons-nous ici au bout de la logique, non seulement de Belle Gloire, mais de la gloire tout court ? Est-ce pour rester fidèle à cette logique que le récit se termine sur une note d'inachèvement? En effet, le conte conclut in media res, avant le combat du roi et des fées. Au-delà de l'explication purement référentielle (Louis XIV n'a pas encore épuisé le champ de ses glorieux exploits à la date où écrit Préchac), l'absence de dénouement tient plutôt à ceci : la logique de la gloire, dont nous venons de voir les limites, n'admet pas d' "Et ils vécurent heureux »...

\section{VI}

Voici donc le récit féerisé de la vie du roi en place : une vie guidée par les défis d'une princesse enchantée, qui, par son nom, par les exploits qu'elle inspire, par son invisibilité aux sujets du roi, sert à renforcer la correspondance étroite entre la fiction et la réalité, malgré le cadre féerique. Certes, l'auteur prend des libertés dans son choix des sujets pour raconter le règne, construisant chaque épisode en fonction d'une issue glorieuse qui n'est due qu'à l'intervention du roi. Mais la logique selon laquelle cette gloire héroïque se construit est directement liée aux enjeux politiques du moment, d'où une insistance sur le danger de la situation et sur la profondeur de la crise, avant l'intervention salvatrice du roi, qui peut laisser le lecteur moderne perplexe. Ainsi, Belle Gloire ne voit dans la crise qu'un potentiel d'héroïsme, et nous lisons les lignes suivantes par rapport à la crise qui correspond à la première partie de la guerre de la Ligue d'Augsbourg: 
Belle Gloire qui apprit que tant de grandes puissances conspiraient contre Sans Parangon, et étaient prêtes à fondre sur ses États, l'en félicita au lieu de le plaindre. (p. 72I)

La félicité glorieuse a donc la même origine que la catastrophe, sans aucun souci de la souffrance occasionnée. En effet, l'insouciance est si totale qu'elle n'est même pas dissimulée. Aussi la princesse annonce-t-elle ouvertement que la crise où se trouve le roi a pour origine sa poursuite de la gloire (les guerres menées, les châteaux construits, etc.) :

songe que tu as plusieurs puissances à combattre, c'est un[e] hydre qui a une infinité de têtes, tes trésors sont épuisés par les complaisances que tu as eues pour moi, au lieu que tes ennemis qui n'ont encore fait aucune dépense, ne manquent ni d'hommes ni d'argent. (p. 722)

En lisant de tels passages, où est poussée à son comble la dignité extravagante qui structure le récit, on a tendance à oublier qu'on a devant soi un éloge de la poursuite louis-quatorzienne de la gloire, et non sa dénonciation. En effet, comment ne pas interpréter ces paroles de la princesse : " tes trésors sont épuisés par les complaisances que tu as eues pour moi ", comme une critique du roi, dans le sillage de la fameuse lettre de Fénelon citée au début du présent article ? Sans doute la logique de Belle Gloire apparaît-elle moins digne qu'extravagante au lecteur moderne ${ }^{\mathrm{II}}$.

Et pourtant, il importe de souligner que la «dignité » dont il est ici question ne correspond pas à notre catégorisation conceptuelle moderne, d'où son opacité à nos yeux. Avant de s'accorder sur l'existence d'une dimension subversive dans le conte, observation fondée sur notre propre lecture du texte, il convient de se pencher sur la réaction des contemporains. Nous savons en fait très peu de choses sur la réception du livre ${ }^{12}$, mais l'absence de scandale est déjà significative. Préchac donne à lire ses contes comme un éloge du pouvoir, et rien n'indique qu'ils n'ont pas été reçus en tant que tel. Si l'on voulait spéculer là-dessus, il vaudrait sans doute mieux se demander, avec Tony Gheeraert, si « peut-être la charge de

II. Voir à cet égard les lectures proposées par Anne Defrance (art. cité, p. 62-65) et Tony Gheeraert (art. cité, p. 683-686).

I2. Le livre paraît en 1698 (Paris, Claude Barbin), et reparât en I724 (Paris, Compagnie des libraires associés), sans la dédicace à la cascade de Marly. Pour une mise en contexte plus large de sa première parution, á l'époque de la mode des contes de fées, on consultera Gevrey, art. cité, p. XXIV-XXIX, sans y trouver, cependant, d'autres indications sur sa réception. 
maître de requêtes que le romancier se verra confier deux ans plus tard est [...] liée à cette performance encomiastique ${ }^{\mathrm{I3}}$ ".

Il faut le souligner : la logique de la gloire - avec ses excès et sa dignité extravagante - n'est pas de l'invention de Préchac qui emprunterait en cela une voie satirique. Elle se trouve partout autour de lui dans l'épanouissement de la légende officielle de Louis le Grand. Ce serait le sujet d'une autre étude que de rapprocher le conte de Préchac des panégyriques académiques du roi, en partant de leur insistance sur le miraculeux et le merveilleux dans l'action du monarque, ainsi que du souci d'ériger des obstacles permettant à la gloire royale d'éclater ${ }^{14}$. Dans notre contexte, il suffira de retourner à la première partie de la guerre de la Ligue d'Augsbourg, pour laquelle l'Académie assumait la fonction de Belle Gloire. Celle-ci se réjouissait, rappelons-nous-en, du fait que " tant de grandes puissances conspiraient contre Sans Parangon, et étaient prêtes à fondre sur ses États ». Or, dans un panégyrique du roi prononcé en 1689 , alors que le pays est en pleine guerre, c'est Paul Tallemant qui adopte pareil discours :

En effet, Messieurs, notre auguste monarque sur la bonne foi des traités vivait dans une tranquillité profonde, cet ordre admirable établi dans tous ses états [...] ne lui laissai $[\mathrm{t}]$ presque plus de nouveaux sujets de gloire à espérer ; et voilà que de toutes parts les ligues formées l'obligent à reprendre les armes, vont lui fournir de nouveaux sujets de victoire, et donner lieu à de nouveaux triomphes ${ }^{15}$.

On reconnaîtra de "Sans Parangon " l'épuisement d'un règne déjà si glorieux qu'il ne reste " presque plus de nouveaux sujets de gloire à espérer ». La félicité devant la menace extérieure inespérée se retrouve quant à elle dans la dernière phrase du panégyrique : "applaudissons-nous sans cesse du bonheur que nous avons de vivre sous un règne si fécond en miracles ${ }^{16}$ ". Ce discours est le dernier panégyrique du roi prononcé au sein de l'Académie. Mais les applaudissements se poursuivent ailleurs, comme on le voit avec "Sans Parangon" de Préchac ${ }^{17}$.

13. Gheeraert, art. cité, p. 670. Ajoutons que Préchac reçoit une gratification de 2000 livres du roi en personne, en 1706, voir Gevrey, art. cité, p. Ix.

I4. Pour une édition moderne, voir Les panégyriques du roi prononcées dans l'Académie française, éd. Pierre Zoberman, Paris, Presses de l'Université de Paris-Sorbonne, I99I.

15. Les panégyriques du roi..., ouvr. cité, p. 243.

I6. Ibid., p. 246.

17. Dans son introduction à ce dernier panégyrique du roi, Zoberman s'intéresse à la disparition du genre, évoquant "une sorte d'épuisement " : "Le nombre de "miracles" a-t-il diminué ? Le discours a-t-il cessé de trouver dans la réalité royale l'air de fiction produit par le merveilleux ? [...] Naturellement, le cérémonial académique continue de pourvoir à la production de l'éloge, mais plus sous cette forme directe et exclusive que constituait le panégyrique du Roi. » (Ouvr. cité, p. 24I) 


\section{VII}

En guise de conclusion, considérons une dernière fois la logique de la gloire, telle qu'elle est inscrite au cœur de l'absolutisme, et au centre de l'univers symbolico-politique de Versailles. Sa place est subrepticement évoquée, sous forme d'une mise en abîme, dans un passage déjà cité du conte de Préchac. Il s'agit de la description du palais enchanté, imaginé par le prince avant sa naissance, et qui anticipe sur sa propre vie :

[...] on passait ensuite dans une grande galerie ornée de glaces et de belles statues de marbre et de bronze, avec des peintures merveilleuses, où l'on remarquait des actions d'un héros si prodigieuses, qu'on ne voyait rien de pareil même dans la fable. (p. 699)

Comment communiquer à travers la peinture « des actions d'un héros si prodigieuses, qu'on ne voyait rien de pareil même dans la fable " ? On sait comment Le Brun a résolu le problème à travers ses " peintures merveilleuses » dans la galerie des Glaces : il représente les actions prodigieuses du héros en les introduisant dans un cadre allégorique où le roi apparaît comme le seul mortel qui revient systématiquement en tête d'un cortège de personnages mythologiques et allégoriques.

La galerie constitue le centre symbolique de Versailles, et par là celui de la France classique tout court. Au centre de ce centre, au milieu du tableau central de la galerie - tableau à considérer, selon Joël Cornette, comme un "document unique, l'image que se faisait le roi de lui-même, de la nature de son pouvoir et de son action ${ }^{18} »-$, nous retrouvons le motif que nous avons étudié tout au long du présent article : le jeune roi qui regarde amoureusement une personnification de la gloire. L'inscription actuelle du tableau semble ne décrire que le geste de la main droite du roi, saisissant le gouvernail de l'État après la mort de Mazarin : "Le Roi gouverne par lui-même, I66I ». Le reste du corps royal, surtout le geste de la main gauche et la direction de son regard (qui ignore l'État qu'il gouverne), en dit pourtant plus long sur la logique de cet absolutisme venant d'être instauré, à l'instar de la première inscription française du tableau par Charpentier : "Louis le Grand dans la fleur de la jeunesse, prend en main le timon de l'État, et renonçant au repos et aux plaisirs, se donne tout entier à l'amour de la véritable gloire ${ }^{\mathrm{I}}$. "

I8. Cornette, ouvr. cité, p. 243.

19. L'inscription originelle de ce tableau, rédigée en latin par Paul Tallemand, est plus proche de la première inscription française dans son insistance sur la gloire (... gloric amore incenditur), voir 
Cette affirmation de la logique absolutiste qui dirige le règne de Sans Parangon ainsi que celui de Louis XIV, trouve sa formulation verbale la plus précise dans un texte où il s'agit précisément, et littéralement, de la transmettre. La gloire constitue, nous l'avons bien vu, "la chose du monde qui [...] est la plus précieuse » au roi. Dans les Mémoires pour l'instruction du Dauphin, nous en trouvons une personnification qui donne à voir sa dignité extravagante, alors qu'elle est présentée comme une mâ̂tresse insatiable. Une autre Belle Gloire, enfantée par celui-là même qui en est l'amant inégalable. C'est dans la péroraison de la première partie des mémoires de l'année I66I que Louis XIV indique ainsi à son fils,

que le réputation ne se peut conserver sans en acquérir tous les jours davantage ; que la gloire enfin n'est pas une maîtresse qu'on puisse jamais négliger, ni être digne de ses premières faveurs, si l'on n'en souhaite incessamment de nouvelles ${ }^{20}$.

Hall Bjørnstad, « "Plus d'éclaircissement touchant la grande galerie de Versailles” : du nouveau sur les inscriptions latines", XVII siècle, $\mathrm{n}^{\circ} 243,6 \mathrm{I}^{\mathrm{e}}$ année, $\mathrm{n}^{\circ}$ 2-2009.

20. Louis XIV, Mémoires pour l'instruction du Dauphin, éd. Pierre Goubert, Paris, Imprimerie Nationale, 1992, p. 59. 